\title{
A special issue on decision intelligence with soft computing
}

\author{
Jie Lu $\cdot$ Guangquan Zhang
}

Published online: 3 September 2009

(C) Springer-Verlag 2009

\section{Introduction}

Decision intelligence brings together traditional business intelligence and decision support systems (DSS) to improve complex, dynamic, uncertain, and un-structured decision problems. Intelligent DSS, along with knowledge-based decision analysis methods, incorporating databases/data warehouses, model bases and intellectual resources, have been well developed. Decision intelligence systems can remove the need to always store data first in a database/data warehouse before it can be used for decision-making. It therefore enables organizations to better understand their dynamic business processes and the factors that affect business performance, which leads to better decisions.

There are a growing number of requirements to deal with the complexity, dynamicity, uncertainty, and un-structure of decision problems. Soft computing, including the techniques of fuzzy logic, rough sets, neural networks, evolutionary computing, swarm intelligence and other similar approaches, have been recently applied in related areas to meet the requirements. Research results show that applying soft computing into decision intelligence effectively improves the intelligent features of the decision process and the quality of decision-making. Some successful applications of these techniques have appeared in various decision problems. Therefore, an updated overview for theoretical research results and practical developments of soft

J. Lu ( $\square) \cdot$ G. Zhang $(\bowtie)$

Faculty of Engineering and Information Technology,

University of Technology, Broadway, PO Box 123,

Sydney (UTS), NSW 2007, Australia

e-mail: jielu@it.uts.edu.au

G. Zhang

e-mail: zhangg@it.uts.edu.au computing in decision intelligence will benefit researchers, businesses, governments, and other organizations.

This special issue provides a high quality forum of the state-of-the-art research and development in line with decision intelligence and soft computing. It was generated from the 2008 International Conference on Intelligent Systems and Knowledge Engineering (ISKE2008, 17-18 November 2008, Xiamen, China) by selecting 9 high quality relevant papers in the scope of the special issue from more than 200 papers in the conference proceedings. The special issue covers both benefits and challenges in this area and draws on the authors' wide-ranging practical experience of strategy development in the field. It provides both a theoretical grounding and practical approaches in the use of soft computing techniques to real-world decision problems, from road safety, breast cancer morbidity, and fashion design to telecommunication customer services. Contributed authors from Australia, Belgium, China, France, Japan, and Spain demonstrate their new research results on how soft computing technologies benefit the development of decision intelligence.

\section{Structure of the special issue}

The nine papers of the special issue can be grouped into two categories: (1) domain-specific developments; (2) general methodology developments of decision intelligence with soft computing.

\subsection{Domain-specific developments}

Five papers in this category present decision intelligence with soft computing in road safety in EU, life situations of senior citizens in Japan, fashion product evaluation in 
France, human brain Tumor classification in Spain, and breast cancer in China.

The first paper by Shen et al. supported by the European Transport Safety Council, presents a hybrid system for road safety performance evaluation. In this hybrid system, a single neuron and a two-layer feedforward network are introduced, with the integration of rough sets techniques, to assign weights to seven road safety performance indicators. The 7 indicators are used to monitor and evaluate the effect of policy interventions, and rank 21 European countries in terms of their road safety. Testing results imply the feasibility of this system and valuable predictive power for the road safety indicators context.

The second paper by Guo reports a novel approach for simplifying the obtained if-then rules from a decision table by the rough sets methods. This approach extracts main features of objects in different decision classes by remarkable degrees. Using the proposed approach, the main features of Japanese industries and the life situations of senior citizens in Japan are analyzed. The analysis results show that the proposed approach have provided deep insight into the situations of objects.

In the third paper, Zeng et al. present a fuzzy multicriteria decision-making method for evaluating fashion products in France. Human perceptions are integrated into the evaluation procedure. Fuzzy set techniques are used to handle linguistic terms in criteria and their weights, as well as the perceptions' explanations for the relationship between sales volumes of products and their components, such as fashion style. By using the proposed method, a set of new fashion products has been well evaluated and ranked.

In the fourth paper by Farias et al., a synergy of advanced signal processing and soft computing strategies is applied to identify different types of human brain tumors. A computational tool is developed which implements the support vector machines and neural networks, and is successfully applied in human brain tumor type classification.

The fifth paper by Li et al. applies fuzzy information diffusion principles to establish an optimal model for risk analysis and decision analysis of breast cancer. It further illustrates a specific process and a successful application of the proposed model and method in breast cancer analysis in Yanpu District, Shanghai, China. The application also shows the effectiveness of this proposed method in dealing with small sample problems, especially in low morbidity cases.

\subsection{General methodology developments}

Four papers in this category effectively and admirably represent the latest results in the development of methods, operators, measures, and algorithms in support decision intelligence.

This sixth paper by Ma et al. proposes a team situation awareness measure method. This method uses utility functions to clarify semantics expressed by linguistic terms. It treats situation awareness as linguistic possibility distributions on a decision object's potential states. Applying this method, team situation awareness can be generated through reasoning and aggregating individual situation awareness based on a hierarchical mental model. A case study of telecoms customer churn explains the effectiveness of the method.

Mining the feature of web Blogs is useful to support product popularity analyzing and business decision-making. The seventh paper deals with Blogger Role mining. To get an appropriate Blogger Role, the study clusters all bloggers' posts and uses the clustering result to revise the Blogger Role. Experiment results on TREC corpus show that Blogger Role explores the characters of bloggers, and mining Blogger Role is useful in product popularity analysis.

The eighth paper by Chen et al. proposes an intelligent decision algorithm based on the particle swarm optimization (PSO) technique, aiming to obtain a feasible floor planning in very large-scale integrated circuit physical placement. Experiments show that the proposed algorithm effectively avoids local minimum and performs well in convergence.

The last paper by Zheng et al. reports recent developments on the robustness of fuzzy schemes in environments with random perturbations. It proposes two methods to analyze the robustness of fuzzy algebraic operators. The effectiveness and features of the methods are shown by simulations. The potential applications in decision intelligence are identified.

Acknowledgments We thank all the authors who have contributed papers to this special issue and all reviewers who helped with reviewing and selecting these papers. We would like to give special thanks to Editor-in-Chief Professor Antonio Di Nola, Co-Editor-inChief Professor Vincenzo Loia and their production team for the continuous support that has made this special issue a great success. 